\title{
Validation of the Arabic version of the Disordered Eating Attitudes in Pregnancy Scale (A-DEAPS) among Lebanese pregnant women: A step closer to Pregorexia Awareness
}

\section{Sarah Gerges}

Holy Spirit University of Kaslik (USEK)

\section{Sahar Obeid}

Holy Spirit University of Kaslik (USEK)

Souheil Hallit ( $\nabla$ souheilhallit@hotmail.com)

Holy Spirit University of Kaslik (USEK)

\section{Research Article}

Keywords: eating disorder, pregorexia, Disordered Eating Attitudes in Pregnancy Scale, Pregnancy, Lebanon

Posted Date: August 20th, 2021

DOl: https://doi.org/10.21203/rs.3.rs-797458/v1

License: (c) (1) This work is licensed under a Creative Commons Attribution 4.0 International License.

Read Full License 


\section{Abstract \\ Background}

Lately, there has been an upsurge in the prevalence of eating disorders (including anorexia, bulimia, orthorexia and recently, pregorexia), mainly due to changes in sociocultural factors. Pregnancy may serve as a propitious basis for the flourishing of "Pregorexia": a notion of popular psychology designating a newly emerging eating disorder. Bannatyne et al. generated a brief pregnancy-specific instrument in furtherance of screening for antenatal eating disorders: the DEAPS (Disordered Eating Attitudes in Pregnancy Scale), which demonstrated a high level of internal consistency and good validity. Our study's objective was to linguistically validate and examine the reliability and psychometric properties of the Arabic version of this previously established pregnancy-specific scale among Lebanese pregnant women.

\section{Methods}

We conceived and implemented a cross-sectional survey between June and July $2021(\mathrm{~N}=433)$. The sample was randomly divided in two as per the SPSS data selection option; the first was used to conduct the DEAPS items factor analysis, whereas the second was used for the confirmatory analysis. Multiple indices of goodness-of-fit were described: the Relative Chi-square ( $\chi 2 / \mathrm{df})$, Root Mean Square Error of Approximation (RMSEA), Tucker Lewis Index (TLI) and Comparative Fit Index (CFI).

\section{Results}

A factor analysis was conducted on Sample $1(\mathrm{~N}=207)$ chosen randomly from the original sample. With the exception of item 8, all other items converged over a two-factor solution (Factor 1: Self-Objectification (Body Control, Body Shame and Esteem) and Factor 2: Pregorexia), explaining a total variance of 39.3\%. In sample $2(\mathrm{~N}=226)$, the one-factor model (Factor 2) that derived from the factor analysis conducted on sample 1, fitted well accordingly to CFI, TLI and $\chi^{2} / \mathrm{df}$ values, but fitted modestly according to RMSEA. The estimates obtained for Models 1 (original scale) and 2 (according to the two-factor solution obtained from the FA in sample 1) fitted less than the third model. The results showed that $28(6.6 \%)$ of the participants were at risk of having disordered eating during pregnancy, whereas $25(5.8 \%)$ had possible presence of disordered eating.

\section{Conclusion}

This study was able to show that the A-DEAPS seems to be a good and reliable tool for the assessment of disordered eating among Lebanese pregnant women.

\section{Introduction}


Lately, there has been an upsurge in the prevalence of eating disorders (including anorexia, bulimia, orthorexia and recently, pregorexia), mainly due to changes in sociocultural factors ${ }^{1}$. Body image disorders are not benign. They dictate risky behaviors that engender major physical and mental health harms, as well as damages to social life ${ }^{1}$. Throughout the history of mankind, the concept of beauty has evolved in a way that nowadays, thinness represents success among women. It is postulated that $15 \%$ of them suffer from body dissatisfaction, thus willing to lose weight ${ }^{2}$.

Although pregnancy being a miraculous process, and despite all the blessings it provides to the mothersto-be, such as becoming parents and acquiring new qualities, it remains, though, a complex "biopsychosocial phenomenon" that witnesses the emergence of concerns about weight, body image, femininity, and self-esteem ${ }^{3}$. Those preoccupations are usually triggered by the pregnancy's emotional hormonal ambivalence, hence inducing new eating disorders or exacerbating previously existing ones, in order to cope with its associated bad feelings as anxiety or even phobic and obsessive-compulsive traits 3 .

Consequently, pregnancy may serve as a propitious basis for the flourishing of "Pregorexia" (also called "Mummyrexia"): a notion of popular psychology designating a newly emerging eating disorder. The term "Pregorexia" first appeared in "The Early Show and Fox" press in 2008, referring to the excessive fear of the pregnancy-induced weight gain, and the drive to control it through various measures; for instance, the extreme restriction of calories intake, excessive exercising, or even diuretics and/or laxatives consumption 4,5 .

This modern nomination results from combining pregnancy + anorexia. Pregorexia is neither considered a medical diagnosis, nor classified in the DSM-5 eating disorders criteria. Additionally, it has not been assigned any international formal or medical definition yet ${ }^{6}$. We might simply define it as anorexia nervosa occurring for the first-time during pregnancy ${ }^{5}$, keeping in mind that "pregorexic" women might experience anorexia and bulimia simultaneously ${ }^{4}$, through a vicious cycle interaction. Some alarming signs: "red flags" include skipping meals, eating alone, and talking about pregnancy as if it is not real (state of denial) ${ }^{4}$. Notably, this phenomenon was also described in "The Perfect Little Bump", a magazine article released far back in 2004, reporting how some pregnant women in New York City were exercising to the stage of pushing their heart rates beyond safe limits, as an apocalyptic resource to minimize their weight gain ${ }^{7}$.

Remarkably, the occurrence of eating disorders (anorexia nervosa, bulimia nervosa, binge eating disorders, eating disorders not otherwise specified...) during pregnancy was of a wide divergence between studies, ranging from $0.6 \%$ to not lesser than $27.8 \%{ }^{8-18}$. These disparities could be explained by the diversity of assessment tools, varying from self-reported questionnaires (based on adapted versions of pre-existing scales for eating disorders, or designed on items derived from the DSM-4 or DSM-5 criteria) to structured interviews ${ }^{9}$, hence emphasizing the need for an international consensual and accurate pregnancy-specific screening tool, as previously suggested by a Delphi study ${ }^{19}$, to make comparisons 
easier in research, and to minimize the probability of false negative and false positive tests. On top of that, a recent systematic review refuted the suitability of the traditional existing measures for evaluating eating disorders in pregnancy, pointing out that among sixteen scales applied across countries, not more than four were presented along with documented psychometric characteristics, yet none was able to stand up for a meritorious level of clinical pertinence (in terms of psychometric performance as internal consistency, criterion-related validity, screening accuracy...) to the point of being set as a "gold standard" measure or substituting the need for a specifically conceived instrument for identifying dysfunctional eating symptoms during pregnancy 20 .

Besides, when it comes to pregorexia, the only documented prevalence worldwide is $5 \%{ }^{21}$. Nevertheless, most healthcare professionals are unaware of this condition ${ }^{6}$. The scarcity of studies exploring this phenomenon proves that raising awareness is essential, considering the importance of a balanced diet during pregnancy and the risks of undernutrition for both the mother-to-be and her fetus, such as miscarriage, low birth weight, type 2 diabetes, cardiovascular diseases, neural tube defects, cognitive disorders, placental abruption, maternal anemia, impaired bone mineralization, post-partum depression, and so forth 4,5 . Barriers to the identification of eating disorders during pregnancy are principally stigma and poor professional training ${ }^{22}$. In addition, the lack of confidential discussions about weight gain, mental health and body dissatisfaction between pregnant women and their physicians accounts for pregorexia's poor detection and management ${ }^{4}$.

Furthermore, literature remarkably highlighted the associations between disordered eating during pregnancy and maternal psychological distress (anxiety, stress, depression) ${ }^{16,23}$, emphasizing the threatening impact of these conditions on mental health. In light of these facts, our role as researchers is only fulfilled when we provide healthcare professionals with an efficient and accurate screening tool for the early detection of pregorexia's cognitive tendencies and toxic behaviors, in order to optimize diagnostic and treatment procedures, hence circumventing fatal repercussions. However, for this sake, it is only in 2019 that Bannatyne et al. generated a brief pregnancy-specific instrument in furtherance of screening for antenatal eating disorders: the DEAPS (Disordered Eating Attitudes in Pregnancy Scale), which demonstrated a high level of internal consistency and good validity ${ }^{17}$, and whose items were selected according to the results of their Delphi study that distinguished disordered symptomatology from the normal anodyne pregnancy-induced changes in eating habits ${ }^{24}$. In order to be applied among Lebanese pregnant women, its cross-cultural adaptation requires a translation process into Arabic (Lebanon's native language). Therefore, our study's objective was to linguistically validate and examine the reliability and psychometric properties of the Arabic version of this previously established pregnancyspecific scale among Lebanese pregnant women.

\section{Methods}

\section{Study Design}


We conceived and implemented a cross-sectional survey between June and July 2021. Online selfadministered questionnaires, established on Google Forms: https://forms.gle/ygHm4Rw4VtdUehSS9, replaced face-to-face structured interviews, making allowance for the risk of interviewers' exposure during the COVID-19 pandemic. Pregnant women aged 18 years and above, and from all Lebanese districts/governorates (Beirut, Mount Lebanon, North Lebanon, South Lebanon, and Bekaa) were invited to take part in this study. They could access the link via social media platforms such as WhatsApp and Facebook applications. Additional participation was guaranteed by the snowball technique, as all participants were requested to share the link among other pregnant women once completing the survey. Their desire for anonymity was respected. Online surveys have been praised for their effectiveness in overcoming geographic distances and easing difficult access to specific populations ${ }^{25}$ (as pregnant women), hence providing a time-saving data collection on a national level.

\section{Minimal sample size calculation}

A minimal sample consisting of 140 pregnant women was a requisite for the validation process, in reference to the recommendations of Comrey and Lee ${ }^{26}$ that ten observations are mandatory for each of the fourteen elements composing the DEAPS.

\section{Translation procedure}

As in this study, the DEAPS was the only scale needing to be validated in Lebanon; its cross-cultural adaptation was initiated by a two-step translation procedure: forward (from English to Arabic), then backward (from Arabic to English), performed by two distinct healthcare professionals. This translation

process is in conformity to international guidelines required for a scale's validation ${ }^{27,28}$. The final version was approved by a committee constituted of the principal investigator, two psychologists and two psychiatrists, and no disparities were found.

\section{Questionnaire and variables}

Our questionnaire was administered in the Arabic language and consisted of closed-ended questions requiring about 15 minutes to be achieved. The first section collected socio-demographic characteristics including age, marital status, place of residence, religion, educational level (complementary, secondary or university), and the household crowding index. The latter was determined by computing the ratio of the number of people living in the respondent's house, to its total number of rooms (except for the kitchen and bathrooms): higher ratios are indicative of lower socioeconomic status ${ }^{29}$. Intensity, duration and frequency of physical activity were assessed to calculate the physical activity index ${ }^{30}$. The second part of the questionnaire included the Disordered Eating Attitudes in Pregnancy Scale (DEAPS): a brief nominal scale, composed of 14 dichotomous items scored as a Yes/No type of answer. In point of fact, researchers have endorsed that physicians should be employing brief and psychometrically satisfactory instruments to detect medical problems in overwhelmed clinical settings ${ }^{31}$, and specifically during antenatal care ${ }^{19}$. The higher the score, the stronger the disordered eating attitudes during pregnancy. The questionnaire also comprised the Dutch Restrained Eating Scale, including 10 items scored on a 5-point 
Likert scale, ranging from $1=$ never to 5 = always. Higher scores reveal higher trends of restrained eating. This scale has been previously validated in Lebanon among adolescents ${ }^{32}$ and adults ${ }^{33}$.

\section{Statistical analysis}

The SPSS software v.25 was used for data analysis. The option "required" was previously set for all questions on Google forms, in order to avoid missing data. Weighting to the general population was done according to the education level. The sample was randomly divided in two as per the SPSS data selection option; the first was used to conduct the DEAPS items factor analysis, whereas the second was used for the confirmatory analysis. A factor analysis was initiated to confirm the legitimacy of the construct of the DEAPS in our sample. The Kaiser-Meyer-Olkin (KMO) value and the Bartlett's sphericity test were checked for sampling adequacy. The factors with Eigen values $>1$ were kept. Reliability was checked using Cronbach's alpha for the total scale and its subscales. The SPSS AMOS software v. 24 was used to conduct confirmatory factor analysis on subsample 2. Multiple indices of goodness-of-fit were described: the Relative Chi-square $(\chi 2 / d f)$ (cut-off values: $<2-5)$, the Root Mean Square Error of Approximation (RMSEA) (close and acceptable fit are considered for values $<0.05$ and $<0.11$ respectively), the Tucker Lewis Index (TLI) and the Comparative Fit Index (CFI) (acceptable values are $\geq 0.90){ }^{34,35}$.

The DEAPS score had a normal distribution since the skewness and kurtosis values varied between - 1 and $+1^{36}$. These conditions consolidate the assumptions of normality in samples larger than $300^{37}$. The Student $t$ test was used to compare two and three or more means respectively, whereas Pearson correlation test was used to correlate two continuous variables. $\mathrm{P}<0.05$ was deemed statistically significant.

\section{Results}

The sample consisted of 433 pregnant women, with a mean age of $28.55 \pm 4.63$ years and a mean weeks of pregnancy of $23.68 \pm 8.68$. Other characteristics and description of the total sample, samples 1 and 2 can be found in Table 1. 
Table 1

Sociodemographic characteristics of the participants.

\begin{tabular}{|llll|}
\hline Variable & $\begin{array}{l}\text { Total sample } \\
(\mathbf{N}=\mathbf{4 3 3})\end{array}$ & $\begin{array}{l}\text { Sample 1 } \\
\mathbf{( N = 2 0 7 )}\end{array}$ & $\begin{array}{l}\text { Sample 2 } \\
\mathbf{( N = 2 2 6 )}\end{array}$ \\
\hline Education level & & & \\
\hline Secondary or less & $62(14.3 \%)$ & $29(14.0 \%)$ & $33(14.6 \%)$ \\
\hline University & $371(85.7 \%)$ & $178(86.0 \%)$ & $193(85.4 \%)$ \\
\hline Marital status & & & \\
\hline Married & $433(100 \%)$ & $207(100 \%)$ & $226(100 \%)$ \\
\hline & Mean $\pm \mathrm{SD}$ & Mean $\pm \mathrm{SD}$ & Mean $\pm \mathrm{SD}$ \\
\hline Age (in years) & $28.55 \pm 4.63$ & $28.43 \pm 4.53$ & $28.66 \pm 4.73$ \\
\hline Household crowding index & $0.82 \pm 0.44$ & $0.81 \pm 0.50$ & $0.83 \pm 0.39$ \\
\hline Physical activity index & $12.08 \pm 14.48$ & $12.46 \pm 13.74$ & $11.72 \pm 15.15$ \\
\hline
\end{tabular}

Factor analysis

A factor analysis was conducted on Sample $1(\mathrm{~N}=207)$ chosen randomly from the original sample. The $\mathrm{KMO}$ value $(=0.804)$ and the $\mathrm{p}$-value of the test of sphericity $(\mathrm{p}<0.001)$ confirmed the sample adequacy. With the exception of item 8 , all other items converged over a two-factor solution (Factor 1: SelfObjectification (Body Control, Body Shame and Esteem) and Factor 2: Pregorexia), explaining a total variance of $39.3 \%$ (Table 2). 
Table 2

Factor analysis of the Disordered Eating Attitudes in Pregnancy Scale (DEAPS) using the promax rotation on sample 1.

DEAPS Item
Factor 1:

Self-Objectification

(Body Control, Body

Shame and Esteem)
Factor 2:

Pregorexia

0.536

2. I have attempted to stop the changes occurring to my body during pregnancy.

3. I have felt anxious about eating in general, or about eating certain foods.

4. I have felt distressed after eating because of its effect on my weight and shape.

5. I have noticed that what I allow myself to eat and how much I can eat is connected to rules and conditions.

6. I felt like there were times when I lost control over my eating and/or body.

0.463

7. I worried that I have, or will, become 'fat' during pregnancy.

8. I have felt distressed and uncomfortably full (i.e., like I am going to burst) after eating a large amount of food.

9. I have spent considerable time researching the most effective ways to minimize how much weight I gain while pregnant.

10. I have spent considerable time researching how I can rapidly lose weight after I have given birth.

11. I have felt disgusted or ashamed with my pregnancy body. $\quad 0.619$

12. My evaluation of my body shape, weight, or size during 0.725 pregnancy has significantly influenced how worthy I believe I am as a mother or person.

13. I have wanted my pregnancy body to be small, like I am "just bump" (i.e., only my stomach appears to have grown, with no weight or shape changes to other areas of my body).

14. I have found myself frequently (at least once a week) comparing my weight, shape, size, or eating habits to other women.

Percentage of variance explained

Cronbach's alpha
11.8

0.771
0.651

0.506
27.5

0.871 
The fit indices of the three tested CFA models are presented in Table 3. As can be seen, the one-factor model (Factor 2) that derived from the factor analysis conducted on sample 1 in this study was fitted well accordingly to $\mathrm{CFI}, \mathrm{TLI}$ and $\chi^{2} / \mathrm{df}$ values, but fitted modestly according to RMSEA. The estimates obtained for Models 1 (original scale) and 2 (according to the two-factor solution obtained from the FA in sample 1) fitted less than the third model.

\section{Table 3}

Fit indices of the three tested confirmatory factor analysis models of the Disordered Eating Attitudes in Pregnancy Scale (DEAPS).

\begin{tabular}{|lllllllll|}
\hline & $\chi^{2}{ }_{(\mathrm{df})}$ & $P$ & TLI & CFI & RMSEA & $90 \% \mathrm{Cl}$ & Factor correlation range \\
\hline Model 1 & $281.06_{(77)}$ & $<.001$ & 0.809 & 0.839 & 0.08 & $0.07-0.09$ & $0.28-0.75$ \\
\hline Model 2 & $132.35_{(64)}$ & $<.001$ & 0.879 & 0.900 & 0.07 & $0.05-0.08$ & $0.40-0.76$ \\
\hline Model 3 & $75.36_{(35)}$ & $<.001$ & 0.904 & 0.926 & 0.07 & $0.05-0.09$ & $0.42-0.75$ \\
\hline $\begin{array}{l}\text { Note: Model 1 = one factor (all items) according to the original validation of the scale; Model 2 }=\text { two } \\
\text { factors (without item 8) according to the factor analysis results obtained in Sample 1; Model 3 = one } \\
\text { factor (10 items) after removing 4 questions not related to pregorexia's eating attitudes during } \\
\text { pregnancy. }\end{array}$ \\
\hline
\end{tabular}

\section{Convergent validity}

Higher A-DEAPS scores correlated well with higher restrained eating scores in the total sample $(r=0.593$; $p<0.001)$.

\section{Correlation of each item of the DEAPS scale with the new total score}

Each item of the DEAPS scale correlated well with the newly generated A-DEAPS total score, with correlation coefficients varying between 0.460 and 0.751 (Table 4). 
Table 4

Correlation of each item of the DEAPS with the newly generated A-DEAPS total score.

Variable

Item-total

correlation

1. I have felt distressed about the changes to my body and/or eating habits during

0.533 pregnancy.

2. I have attempted to stop the changes occurring to my body during pregnancy.

0.583

3. I have felt anxious about eating in general, or about eating certain foods.

0.460

4. I have felt distressed after eating because of its effect on my weight and shape.

0.730

5. I have noticed that what I allow myself to eat and how much I can eat is connected to $\quad 0.510$ rules and conditions.

7. I worried that I have, or will, become 'fat' during pregnancy.

0.751

9. I have spent considerable time researching the most effective ways to minimize how $\quad 0.682$ much weight I gain while pregnant.

10. I have spent considerable time researching how I can rapidly lose weight after I have $\quad 0.529$ given birth.

13. I have wanted my pregnancy body to be small, like I am "just bump" (i.e., only my stomach appears to have grown, with no weight or shape changes to other areas of my body).

14. I have found myself frequently (at least once a week) comparing my weight, shape, size, or eating habits to other women.

$\mathrm{P}<0.001$ for all correlations.

\section{Rate of disordered eating during pregnancy among our sample}

Based on the previous work of Barthels et al. ${ }^{38}$, we considered the 90th and 95th percentile as the cutoff points for being at risk of disordered eating and possible presence of disordered eating during pregnancy, respectively. The results showed that $28(6.6 \%)$ of the participants were at risk of having disordered eating during pregnancy, whereas $25(5.8 \%)$ had possible presence of disordered eating.

\section{Bivariate analysis}

The results showed that no significant difference was seen in terms of A-DEAPS scores between Christian and Muslim pregnant women ( $3.95 \pm 2.63$ vs $4.10 \pm 2.81 ; p=0.666)$, and between those having a university vs secondary or less level of education ( $4.03 \pm 2.76$ vs $4.24 \pm 2.89 ; p=0.539)$. Furthermore, lower A-DEAPS scores were significantly but weakly correlated with higher household crowding index $(r=-0.195 ; p<0.001)$, but not with age $(r=0.011 ; p=0.823)$.

\section{Discussion}




\section{Factorial Validity - Finding Support for the One-Factor Model}

In this paper, we inspected the psychometric properties of the Arabic version of the DEAPS in a sample of Lebanese pregnant women; this would help surmount language barriers and properly screen for pregorexia. Within the literature, the authors who developed the DEAPS ${ }^{17}$ suggested a one-factor model for the scale. Within this study, three competing measurement models were tested; the CFA of the original model showed poor fitting results. When removing item 8 that rather corresponds to stress-binging, and items 6,11 and 12 (factor 1 ; see Table 2) that are more related to objectified body consciousness (selfobjectification) including body image control, body shame, and self (body)-esteem ${ }^{39}$ (not related to pregorexia's disordered eating attitudes per se), the results came out to be more satisfactory. Thus, we have provided evidence that the one-factorial model that includes 10 items only, is the one that should be considered regarding the DEAPS.

\section{Internal Consistency and Convergent Validity - Further Support for the Model}

The 10-item, one-factor model seems to be internally consistent, and the reliability analysis of those 10 items turned out to be very good as well $(a=0.871)$. This value was similar to the one obtained in the original version of the DEAPS $(\alpha=0.85)$. The item-total correlation appeared to be good as well, suggesting that the chosen items represent the A-DEAPS well. Furthermore, the A-DEAPS correlated well with the restrained eating score, demonstrating the convergent validity of the scale.

\section{Prevalence rates of disordered eating in pregnancy among our sample}

Our findings revealed that within our sample, drawn from the Lebanese population, $5.8 \%$ of respondents were going through their pregnancies while most probably burdened with pregorexia-related eating attitudes, in consistence with the previously reported prevalence of $5 \%{ }^{21}$, and those detected among samples of pregnant women in Western countries: by Turton et al. (scores above the Eating Attitudes Test cut-off: $4.9 \%$ ) in UK ${ }^{12}$, Easter et al. (anorexia nervosa, bulimia nervosa, purging disorders and eating disorders not otherwise specified: $5.7 \%$ ) in UK as well ${ }^{15}$, and Pettersson et al. (eating restraint, and shape, weight, and eating concerns: $5.3 \%$ ) in Sweden ${ }^{13}$. Conversely, this prevalence rate does not parallel the $22 \%$ recorded by Bannatyne et al. when applying a cutoff point of 8 (different from ours) to their originally developed scale (14 items, versus the 10 items retained in the A-DEAPS) ${ }^{17}$. Thus, to decide on this issue, setting a cut-off point, in conformity with a blinded-specialist's diagnosis should interest future assessments of the A-DEAPS. Additionally, $6.6 \%$ were vulnerable to suffer from eventual disordered eating. In total, a considerable number of participants was prone to a dreadful physical and psychological condition throughout pregnancy, and to all the adverse outcomes demonstrated to come along, increasing the need for health care. Through this study, we thus emphasized the vitalness of the implementation of systematic antenatal screening programs in Lebanon, for which we provided an adequate tool, without neglecting the necessity of further diagnosis and monitoring, when deemed necessary. 
Relations to Socio-Demographic Variables - household crowding index was the only variable associated with disordered eating in pregnant women

Our results showed that higher household crowding index (reflecting a lower socioeconomic status) was significantly associated with lower A-DEAPS scores, in line with a previous study conducted in Lebanon among the general population ${ }^{40}$. People with low income have less healthy dietary habits since their main priority relies in the price of the food items, and their lower priority being for health. Moreover, the severe economic/financial crisis that is currently hitting Lebanon, does not give people the luxury of buying the food they want.

\section{Limitations}

The cross-sectional design does not allow to draw causative associations. The symptoms of disordered eating were self-reported by participants and not clinically diagnosed by a healthcare professional, making our results susceptible to a possible information bias. The snowball technique followed during the data collection process predisposes us to a selection bias. Residual confounding bias is possible since other factors associated with disordered eating (e.g. mental health issues, smoking, etc.) were not taken into account in this paper.

\section{Conclusion}

This study was able to show that the A-DEAPS seems to be a good and reliable tool for the assessment of disordered eating among Lebanese pregnant women. Further research should aim at validating this scale in the clinical setting and among other Arabic-speaking populations.

\section{Declarations}

\section{Ethical approval and consent to participate:}

The Psychiatric Hospital of the Cross Ethics and Research Committee approved this study protocol (HPC014-2021). The survey's introductory section detailed the study's objectives. Approving a statement at the beginning of the questionnaire: "I consent to participate in this study" (by answering "yes") allowed participants moving to the next sections. Thus, it was considered equal to a written informed consent. All methods were performed in accordance with the relevant guidelines and regulations (Declaration of Helsinki).

Consent to publish: not applicable.

Availability of data and materials: The datasets generated and/or analysed during the current study are not publicly available due to restrictions from the ethics committee but are available from the corresponding author on reasonable request.

Competing interests: The authors have no conflicts of interest to report. 
Funding: None.

Authors' contributions: SO and SH conceived and designed the survey. SH involved in the statistical analysis and data interpretation. SG wrote the manuscript. All authors read the manuscript, critically revised it for intellectual content, and approved the final version.

Acknowledgments: The authors would like to thank all participants.

\section{Abbreviations}

DEAPS

Disordered Eating Attitudes in Pregnancy Scale

RMSEA

Root Mean Square Error of Approximation

TLI

Tucker Lewis Index

$\mathrm{CFI}$

Comparative Fit Index

\section{References}

1. Rzońca E, Bień A, Iwanowicz-Palus G. Zaburzenia odżywiania - problem wciąż aktualny = eating disorders - an ongoing problem. Journal of education, health and sport. 2016;6(12):267-273.

2. Médici GM, Haga SR, Pinheiro MA, Casseb GRM, Colucci RF, de Brito Barbosa L. Transtornos alimentares na pós modernidade: Vigorexia, pregorexia e ortorexia. International Journal of Nutrology. Sep 2018;11(S 01).

3. Bjelica A, Cetkovic N, Trninic-Pjevic A, Mladenovic-Segedi L. The phenomenon of pregnancy - a psychological view. Ginekol Pol. 2018;89(2):102-106.

4. Mathieu J. What is pregorexia? J Am Diet Assoc. 2009;109(6):976-979.

5. Mandera A, Pawlikowska A, Szuster EM, Całkosiński A, Kostrzewska P, Majewski M. The pregorexia anorexia during the pregnancy. Journal of Education. 2019;9(5):137-144.

6. Tuncer E, Gumus AB, Keser A. The importance of pregorexia awareness. Clinical and Experimental Health Sciences. 2020;10(3):186-190.

7. Abraham L. The perfect little bump. New York Magazine- Pregnant and Still Thin- Nymag Web site. https://nymag.com/nymetro/health/features/9909/. Updated 2004. Accessed Jul 20, 2021.

8. Soares RM, Nunes MA, Schmidt MI, et al. Inappropriate eating behaviors during pregnancy: Prevalence and associated factors among pregnant women attending primary care in southern brazil. The International journal of eating disorders. 2009;42(5):387-393.

9. Bye A, Nath S, Ryan EG, et al. Prevalence and clinical characterisation of pregnant women with eating disorders. European eating disorders review. 2020;28(2):141-155. 
10. Watson HJ, Von Holle A, Hamer RM, et al. Remission, continuation and incidence of eating disorders during early pregnancy: A validation study in a population-based birth cohort. Psychological medicine. 2013;43(8):1723-1734.

11. FAIRBURN CG, STEIN A, JONES R. Eating habits and eating disorders during pregnancy. Psychosomatic medicine. 1992;54(6):665-672.

12. Turton $P$, Hughes $P$, Bolton $H$, Sedgwick P. Incidence and demographic correlates of eating disorder symptoms in a pregnant population. The International journal of eating disorders. 1999;26(4):448452.

13. Pettersson C, Zandian M, Clinton D. Eating disorder symptoms pre- and postpartum. Arch Womens Ment Health. 2016;19(4):675-680.

14. Kelly RH, Zatzick DF, Anders TF. The detection and treatment of psychiatric disorders and substance use among pregnant women cared for in obstetrics. The American journal of psychiatry. 2001;158(2):213-219.

15. Easter A, Bye A, Taborelli E, et al. Recognising the symptoms: How common are eating disorders in pregnancy? European eating disorders review. 2013;21(4):340-344.

16. Santos AMD, Benute GRG, Santos NOD, Nomura RMY, de Lucia, Mara Cristina Souza, Francisco RPV. Presence of eating disorders and its relationship to anxiety and depression in pregnant women. Midwifery. 2017;51:12-15.

17. Bannatyne AJ. Disordered eating in pregnancy: The development and validation of a pregnancyspecific screening instrument. Bond University; 2019.

18. Broussard B. Psychological and behavioral traits associated with eating disorders and pregnancy: A pilot study. Journal of midwifery \& women's health. 2012;57(1):61-66.

19. Bannatyne A, Hughes R, Stapleton P, Watt B, MacKenzie-Shalders K. Consensus on the assessment of disordered eating in pregnancy: An international delphi study. Arch Womens Ment Health. 2018;21(4):383-390.

20. Bannatyne AJ, McNeil E, Stapleton P, MacKenzie-Shalders K, Watt B. Disordered eating measures validated in pregnancy samples: A systematic review. Eating disorders. 2019:1-26.

21. Babicz-Zielińska E, Wądołowska L, Tomaszewski D. Eating disorders: Problems of contemporary civilisation - A review. Pol J Food Nutr Sci. 2013;63(3):133-146.

22. Bye A, Shawe J, Bick D, Easter A, Kash-Macdonald M, Micali N. Barriers to identifying eating disorders in pregnancy and in the postnatal period: A qualitative approach. BMC Pregnancy Childbirth. 2018;18(1):114.

23. Easter A, Solmi F, Bye A, et al. Antenatal and postnatal psychopathology among women with current and past eating disorders: Longitudinal patterns. European eating disorders review. 2015;23(1):1927.

24. Bannatyne AJ, Hughes R, Stapleton P, Watt B, MacKenzie-Shalders K. Signs and symptoms of disordered eating in pregnancy: A delphi consensus study. BMC pregnancy and childbirth. 2018;18(1):262. 
25. Wright KB. Researching Internet-Based populations: Advantages and disadvantages of online survey research, online questionnaire authoring software packages, and web survey services. Journal of computer-mediated communication. 2005;10(3):00.

26. Comrey AL, Lee HB. A first course in factor analysis. Psychology press; 2013.

27. Beaton DE, Bombardier C, Guillemin F, Ferraz MB. Guidelines for the process of cross-cultural adaptation of self-report measures. Spine (Philadelphia, Pa. 1976). 2000;25(24):3186-3191.

28. Beaton D, Bombardier C, Guillemin F, Ferraz MB. Recommendations for the cross-cultural adaptation of health status measures. new york: American academy of orthopaedic surgeons. New York: American Academy of Orthopaedic Surgeons. 2002:12: 1-9.

29. Melki IS, Beydoun HA, Khogali M, Tamim H, Yunis KA. Household crowding index: A correlate of socioeconomic status and inter-pregnancy spacing in an urban setting. Journal of Epidemiology and Community Health. 2004;58:476-480.

30. Weary-Smith KA. Validation of the physical activity index (PAI) as a measure of total activity load and total kilocalorie expenditure during submaximal treadmill walking. University of Pittsburgh; 2007.

31. Marquer $C$, Barry $C$, Mouchenik Y, et al. A rapid screening tool for psychological distress in children 3-6years old: Results of a validation study. BMC psychiatry. 2012;12(1):170.

32. Boulos Nakhoul T, Mina A, Soufia M, Obeid S, Hallit S. Restrained eating in lebanese adolescents: Scale validation and correlates. BMC pediatrics. 2021;21(1):257.

33. Saade S, Hallit S, Haddad C, et al. Factors associated with restrained eating and validation of the arabic version of the restrained eating scale among an adult representative sample of the lebanese population: A cross-sectional study. Journal of eating disorders. 2019;7(1):24.

34. Byrne BM. Structural equation modeling with mplus: Basic concepts, applications, and programming. Routledge; 2013.

35. Marsh HW, Hau K, Wen Z. In search of golden rules: Comment on hypothesis-testing approaches to setting cutoff values for fit indexes and dangers in overgeneralizing hu and bentler's (1999) findings. Structural equation modeling. 2004;11(3):320-341.

36. Hair JJ, Hult G, Ringle C, Sarstedt M. A primer on partial least squares structural equation modeling (PLS-SEM). SAGE publications; 2016.

37. Mishra P, Pandey C, Singh U, Gupta A, Sahu C, Keshri A. Descriptive statistics and normality tests for statistical data. Annals of cardiac anaesthesia. 2019;22(1):67-72.

38. Barthels F, Meyer F, Pietrowsky R. Die düsseldorfer orthorexie Skala-Konstruktion und evaluation eines fragebogens zur erfassung ortho-rektischen ernährungsverhaltens. Zeitschrift für klinische Psychologie und Psychotherapie (Göttingen, Germany). 2015;44(2):97-105.

39. McKinley NM, Hyde JS. THE OBJECTIFIED BODY CONSCIOUSNESS SCALE development and validation. Psychology of Women Quarterly. 1996;20(2):181-215. 
40. Haddad C, Khoury C, Salameh P, et al. Validation of the arabic version of the eating attitude test in lebanon: A population study. Public Health Nutrition:1-12. 